\title{
Maternal Caffeine Intake Disrupts Eggshell Integrity and Retards Larval Development by Reducing Yolk Production in a Caenorhabditis elegans Model
}

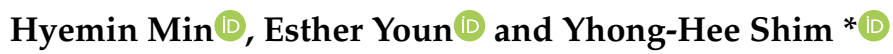 \\ Department of Bioscience and Biotechnology, Konkuk University, Seoul 05029, Korea; \\ mintmin@konkuk.ac.kr (H.M.); dptmej@konkuk.ac.kr (E.Y.) \\ * Correspondence: yshim@konkuk.ac.kr; Tel.: +82-2-450-4059; Fax: +82-2-455-9956
}

Received: 7 April 2020; Accepted: 6 May 2020; Published: 7 May 2020

\begin{abstract}
During pregnancy, most women are exposed to caffeine, which is a widely consumed psychoactive substance. However, the consequences of maternal caffeine intake on the child remain largely unknown. Here, we investigated the intergenerational effects of maternal caffeine intake on offspring in a Caenorhabditis elegans model. We treated a young mother (P0) with $10 \mathrm{mM}$ of caffeine equivalent to $2-5$ cans of commercial energy drinks and examined its reproduction and growth rate from $\mathrm{P} 0$ to $\mathrm{F} 2$ generation. The fertility decreased and embryonic lethality increased by defective oocytes and eggshell integrity in caffeine-ingested mothers, and F1 larval development severely retarded. These results were due to decreased production of vitellogenin protein (yolk) in caffeine-ingested mothers. Furthermore, effects of RNA interference of vitellogenin (vit) genes, vit-1 to vit-6, in P0 mothers can mimic those by caffeine-ingested mothers. In addition, RNA interference (RNAi) depletion of unc-62 (human Meis homeobox), a transcriptional activator for vit genes, also showed similar effects induced by caffeine intake. Taken together, maternal caffeine intake reduced yolk production mediated by the UNC-62 transcription factor, thereby disrupting oocyte and eggshell integrity and retarding larval development. Our study suggests the clinical significance of caffeine intake for prospective mothers.
\end{abstract}

Keywords: caffeine; 1,3,7-trimethylxanthine; maternal effect; intergenerational effect; reproduction; yolk protein; vitellogenin; UNC-62; eggshell integrity; Caenorhabditis elegans

\section{Introduction}

Caffeine is the most widely consumed bioactive molecule and its consumption has been increasing worldwide. A clinical issue about caffeine intake during pregnancy is emerging owing to the possible adverse impact of maternal nutritional status on child development [1,2]. However, the mechanism by which information is shared between the mother and child remains largely unknown.

Caenorhabditis elegans is an excellent animal model to study intergenerational effects of nutrient intake on the progeny because it is easy to examine embryonic and post-embryonic developmental processes in a large population of progeny at the organismal level. Furthermore, owing to its relatively small genome sequence and systematic phenotypic analyses, each process can be assessed at the molecular level as well [3,4]. C. elegans is also an excellent animal model to study reproduction because this hermaphrodite contains both egg and sperm. Thus, the entire reproductive progress from mitosis and meiosis of germ cells, and gametogenesis can be observed in one gonad arm. After the production of egg and sperm, the process of fertilization and even early embryogenesis are possibly observed simultaneously [5]. Several studies on caffeine intake in the C. elegans model have shown both beneficial and adverse effects on $C$. elegans development depending on the intake dose. At a high dose of caffeine 
(30 $\mathrm{mM})$, stress responses were induced, larval development was inhibited, and even food-avoidance behavior was elicited when fed at the early larval stage of C. elegans [6-8]. However, at doses $<10 \mathrm{mM}$ of caffeine, the life span of $C$. elegans was extended $[9,10]$. Recent studies also suggest that caffeine has neuroprotective effects [11].

In this study, we examined reproduction in a caffeine-ingested mother, and consequently its embryonic and larval development in a C. elegans model. We found that $10 \mathrm{mM}$ of caffeine intake caused defects in oocytes, increased embryonic lethality, and larval growth retardation. We further investigated the underlying molecular mechanisms and found that expression of unc-62 gene (human Meis homeobox transcription factor) and its target genes, vit (vitellogenin) genes, was severely reduced. This resulted in defects in oocytes and intergenerational effects, including disrupted eggshell integrity and further retardation in larval development. Taken together, the results of this study suggest that caffeine intake by the mother can affect development of the progeny due to the reduction in yolk protein, which is a major source of nutrients in C. elegans oocytes and embryos.

\section{Materials and Methods}

\subsection{Caenorhabditis Elegans Strains and Caffeine Treatment}

Caenorhabditis elegans strains were maintained at either 15 or $20^{\circ} \mathrm{C}$ on nematode growth medium (NGM) agar plates seeded with Escherichia coli strain OP50, as described previously [12]. The following strains were used in the present study: N2 (C. elegans wild isolate, Bristol variety), RB1982: vit-1(ok2616) X, RB2365: vit-2(ok3211) X, RB2382: vit-5(ok3239) X, DH1033: bIs1 (vit-2::GFP+rol-6(su10060)) X, and BC12843: dpy-5(e907) I; sIs11286(rCesK07H8.6(vit-6)::GFP+pCeh361). To examine the effects of caffeine intake, caffeine (Sigma-Aldrich, St. Louis, MO, USA) was added to NGM before autoclaving to obtain final concentrations of 5, 10, and $30 \mathrm{mM}$ caffeine. Synchronized L4-stage animals were exposed to caffeine for $24 \mathrm{~h}$ at $20^{\circ} \mathrm{C}$ and then the adult-stage mothers and their progenies were examined.

\subsection{Analysis of the Number of Progenies, Embryonic Lethality, and Percent Larval Development}

L4-stage wild-type N2 hermaphrodites were individually cloned onto either caffeine-containing (5-30 mM) or caffeine-free $(0 \mathrm{mM}) \mathrm{NGM}$ agar plates and grown at $20^{\circ} \mathrm{C}$. They were transferred to new plates in $24 \mathrm{~h}$ intervals for 4 days to allow embryo production. Laid embryos were considered dead if they did not hatch after $48 \mathrm{~h}$ at $20{ }^{\circ} \mathrm{C}$. The number of progenies was calculated as the total number of non-hatched and hatched embryos produced by a single mother. Embryonic lethality was calculated as the percentage of non-hatched embryos of the total number of embryos produced for 4 days. Percent larval development was calculated as the percentage of larvae of the total number of hatched embryos that reached each developmental stage, as previously described [6]. We distinguished the developmental larval stages as follows: L1, the smallest larvae $<0.3 \mathrm{~mm}$; L2, larvae larger than L1 (body length, 0.3-0.4 mm) but with no characteristics of L3; L3, larvae with a white spot in the vulva region (body length, $0.4-0.6 \mathrm{~mm}$ ); L4, larvae with a characteristic half-moon-like shape in the vulva region (body length, $0.6-0.8 \mathrm{~mm}$ ); adults, animals with an opened vulva with eggs in the uterus.

\subsection{Western Blot Analysis}

Western blot analysis was performed using whole animal protein extract obtained from ca. 200 gravid adult hermaphrodites of each condition per gel well. Antibodies bound to a nitrocellulose membrane (PROTRAN BA83, Whatman, Sigma-Aldrich, St. Louis, MO, USA) were visualized with Chemiluminescence (ECL) Western blotting detection kit (Amersham, GE Healthcare Life Sciences, Pittsburgh, PA, USA), and the respective band intensities were measured with LAS-3000 image analyzer using Multi Gauge software (v.3.0, Fuji Film, Tokyo, Japan). The following primary and secondary antibodies were used: rabbit anti-GFP (1:1000, Novus, St. Charles, MO, USA), mouse anti- $\alpha$-tubulin (1:1000; Sigma-Aldrich, St. Louis, MO, USA), HRP-conjugated goat anti-rabbit IgG (1:1000; Santa 
Cruz Biotechnology, Dallas, TX, USA), and HRP-conjugated donkey anti-mouse IgG (1:1000; Jackson ImmunoResearch, PA, USA).

\subsection{Analysis of Oocyte and Eggshell Integrity}

To investigate oocyte and eggshell integrity after caffeine intake, membrane permeability was assessed using FM4-64 dye (Sigma-Aldrich, St. Louis, MO, USA), as previously described [13]. In brief, caffeine-ingested mothers were dissected in $150 \mathrm{mM} \mathrm{KCl}$ with $30 \mu \mathrm{M}$ of FM4-64 dye to observe oocytes and embryos. The proportion of either embryos or oocytes infiltrated by FM4-64 was measured using a Zeiss microscope at $40 \times$ magnification. For each case, three independent experiments were performed.

\subsection{RNA Interference (RNAi) Assays}

RNAi experiments were performed using the soaking method, as previously described [14]. dsRNAs of vit-1, vit-2, vit-3, vit-4, vit-5, vit-6, and unc-62 genes were synthesized in vitro using the respective cDNA template. The cDNA templates flanked by T7 promoter sequences were generated by PCR using T7 primer, 5'-GTAATACGACTCACTATAGGGC-3' and CMo422 primer, 5'-GCGTAATACGACTCACTATAGGGAACAAAAGCTGGAGCT-3'. Soaking buffer without dsRNA was used as the negative mock RNAi control. L4-stage animals were soaked in dsRNA solution for $24 \mathrm{~h}$, then transferred onto caffeine-containing NGM agar plates to grow for $24 \mathrm{~h}$ until the animals reached the adult stage. The adult-stage animals were evaluated by membrane integrity assay.

\subsection{DNA Staining in Oocytes}

To observe whether oocytes of caffeine-ingested mothers have six pairs of homologous chromosomes (bivalents), DNA staining was performed, as previously described [14]. Animals were dissected to extrude gonads in $10 \mu \mathrm{L}$ of M9 buffer containing $100 \mu \mathrm{g} / \mathrm{mL}$ tetramisole on a poly-L-lysine-coated slide, covered with a coverslip, freeze-cracked with liquid nitrogen, and fixed with cold methanol and cold acetone. The specimens were then stained with $1 \mu \mathrm{M}$ TO-PRO-3 (Molecular Probes, Eugene, OR, USA) for $1 \mathrm{~h}$ at $20^{\circ} \mathrm{C}$ to stain DNA and then observed under a confocal microscope (Olympus, FV1000 Spectral, Tokyo, Japan).

\subsection{Real Time RT-PCR ( $q R T-P C R)$}

Adult hermaphrodites of wild-type that were treated or not treated with caffeine $(10 \mathrm{mM})$ were collected in TRIzol (Invitrogen, Waltham, MA, USA), and total RNA was extracted using a phase lock gel (MaXtract High Density, Qiagen, Germantown, MD, USA). cDNA was synthesized using oligo-dT primer and M-MLV reverse transcriptase (Invitrogen, Waltham, MA, USA). qRT-PCR assays were performed using SYBR Green PCR Master Mix (Applied Biosystems, Waltham, MA, USA). The final PCR volume was $10 \mu \mathrm{L}$. act-1 mRNA was used as an endogenous control for data normalization. The primers used for the measurement of expression of the unc-62 gene were as follows: forward, 5'-TAAGACATACCCAAGAGAATGCTG-3' and reverse, 5' -TTTGCCTTTCAGACAGACCA-3'.

\subsection{Statistical Analysis}

All experiments were repeated more than three times for statistical evaluation of the data. Two-tailed Student's $t$-test was used to calculate $p$-values; $p<0.05$ was considered significant. The data are expressed as the mean \pm standard deviation (SD). 


\section{Results}

3.1. Maternal Caffeine Intake Causes a Reduction in Fertility and Retardation in the Developmental Growth of Progeny in C. elegans

To investigate whether caffeine intake by the mother has intergenerational effects on offspring, we fed caffeine only to P0 mothers, as shown in Figure 1, and measured the fertility and the developmental growth of offspring. It has been previously reported that the effects of caffeine treatment are dose dependent [6]. Therefore, we first examined the effect of doses of caffeine intake on reproduction by feeding $0,5,10$, and $30 \mathrm{mM}$ of caffeine to hermaphrodites of wild-type L4-stage animals for $24 \mathrm{~h}$. The number of progenies was significantly decreased and embryonic lethality was increased when mothers were fed 10 or $30 \mathrm{mM}$ of caffeine (Figure 2A,B). These results indicate that $>10 \mathrm{mM}$ of caffeine intake seriously reduced fertility. In this study, the effects of $10 \mathrm{mM}$ of caffeine intake were examined in the subsequent experiments because some mothers fed $30 \mathrm{mM}$ of caffeine became sick.

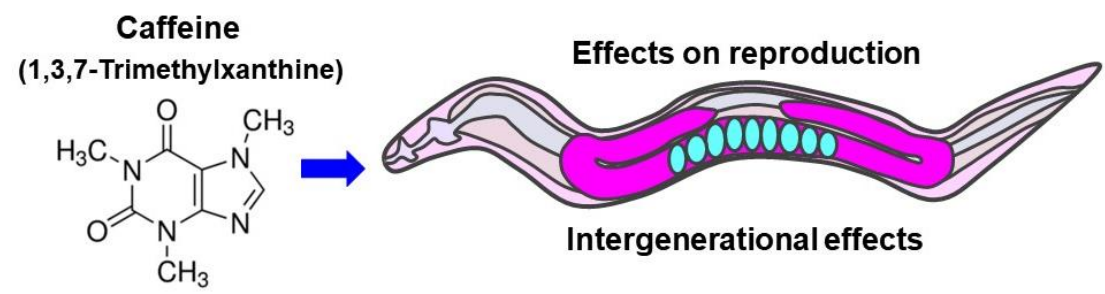

Figure 1. Caenorhabditis elegans P0 mothers were fed single compound caffeine (1,3,7-trimethylxanthine).

To determine whether caffeine intake by the mother has intergenerational effects, fertility including the number of progenies and embryonic lethality was assessed from P0 to F2 generation in C. elegans (Figure 2C). The number of progenies decreased in P0 mothers fed $10 \mathrm{mM}$ caffeine, but not in F1 and F2 generation mothers. In addition, embryonic lethality increased in F1 embryos produced by the caffeine-ingested P0 mother, but not in F2 and F3 embryos (Figure 2D,E). These results suggest that caffeine intake reduced fertility in P0 mothers, but not in F1 or F2 mothers.

Next, we tested the possibility that caffeine intake could affect growth of hatched offspring produced by caffeine-ingested mothers. We evaluated the developmental growth rate in F1 and F2 generations of caffeine-ingested P0 mothers. Interestingly, caffeine-ingested P0 mothers showed a significantly retarded developmental growth rate in the F1 generation (Figure 3A). However, in the F2 generation, no growth retardation was observed (Figure 3B). These results suggest that caffeine intake by the mother delays growth of the subsequent F1 generation (ca. 70\% adult without caffeine intake, but $0 \%$ adult with caffeine at $72 \mathrm{~h}$ growth), but not of the $\mathrm{F} 2$ generation (ca. $70 \%$ adult in both $0 \mathrm{mM}$ and $10 \mathrm{mM}$ caffeine intake at $72 \mathrm{~h}$ growth). 
A

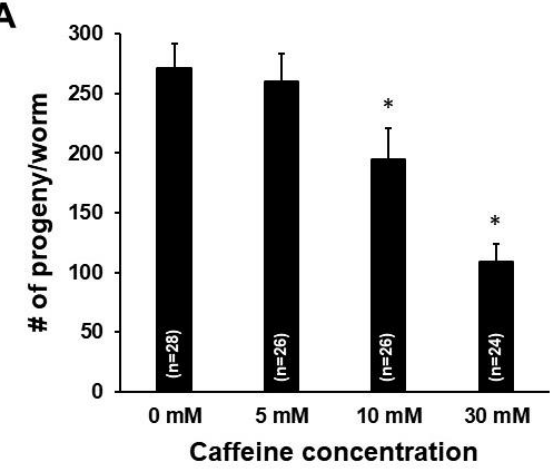

C Po
B

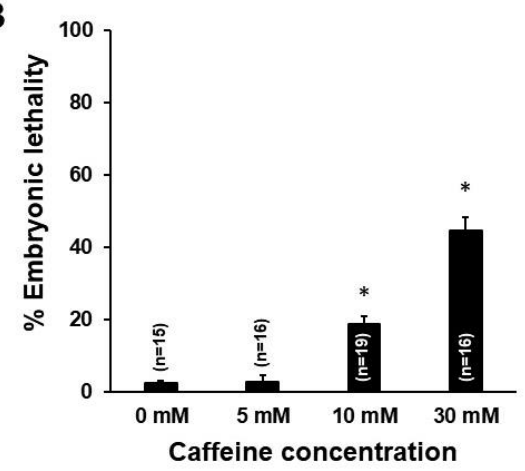

F2
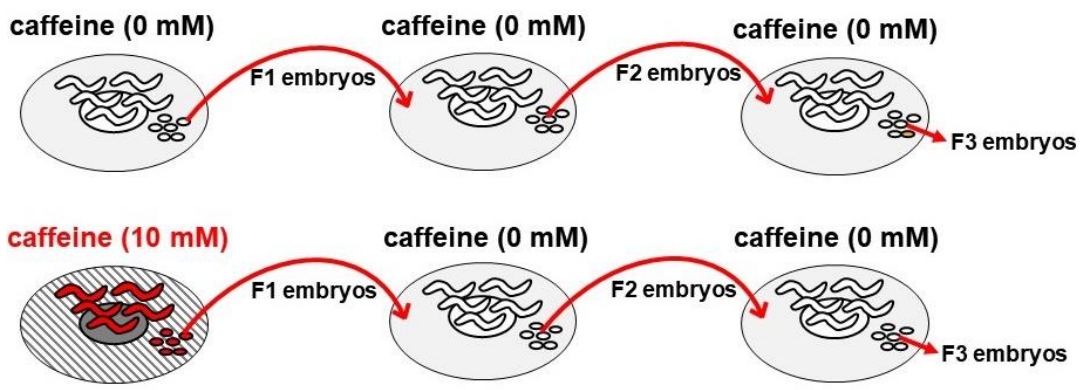

D

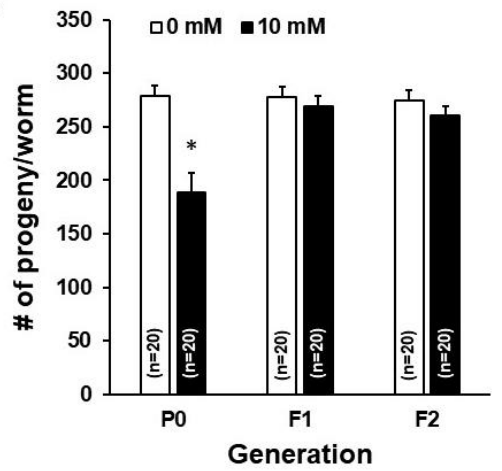

$\mathbf{E}$

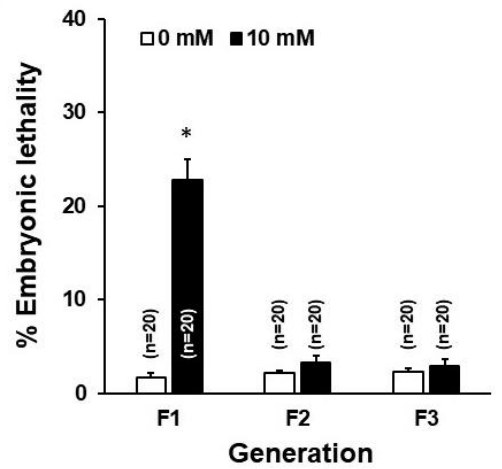

Figure 2. Caffeine intake caused a reduction in fertility of $\mathrm{P} 0$ mothers and an increase in $\mathrm{F} 1$ embryonic lethality in Caenorhabditis elegans. (A) Total number of progenies by caffeine-ingested mothers $(5,10$, and $30 \mathrm{mM}$ ) compared to mothers with a caffeine-free diet $(0 \mathrm{mM}) .{ }^{*} p<0.05$. (B) The percentage of embryonic lethality among the total number of progenies produced by caffeine-ingested $\mathrm{P} 0$ mothers that were fed 0,5 , 10 , and $30 \mathrm{mM}$ of caffeine at the L4 stage for $24 \mathrm{~h} .{ }^{*} p<0.05$. (C) A scheme of assays for intergenerational effects of caffeine intake by $\mathrm{P} 0$ mothers. The P0 mothers were fed $10 \mathrm{mM}$ of caffeine, and reproduction and growth were measured from $\mathrm{P} 0$ to F2 generation for analysis of intergenerational effects of caffeine intake by P0 mothers. (D) The total number of progenies produced by caffeine-ingested P0 mothers and their offspring (black bars) compared to caffeine-free diet P0 mothers and their offspring (white bars). ${ }^{*} p<0.05$. (E) The percentage of embryonic lethality among the total number of progenies produced by caffeine-ingested P0 mothers and their offspring (black bars), and caffeine-free diet P0 mothers and their offspring (white bars). Error bars represent SD. ${ }^{*} p<0.05$. 

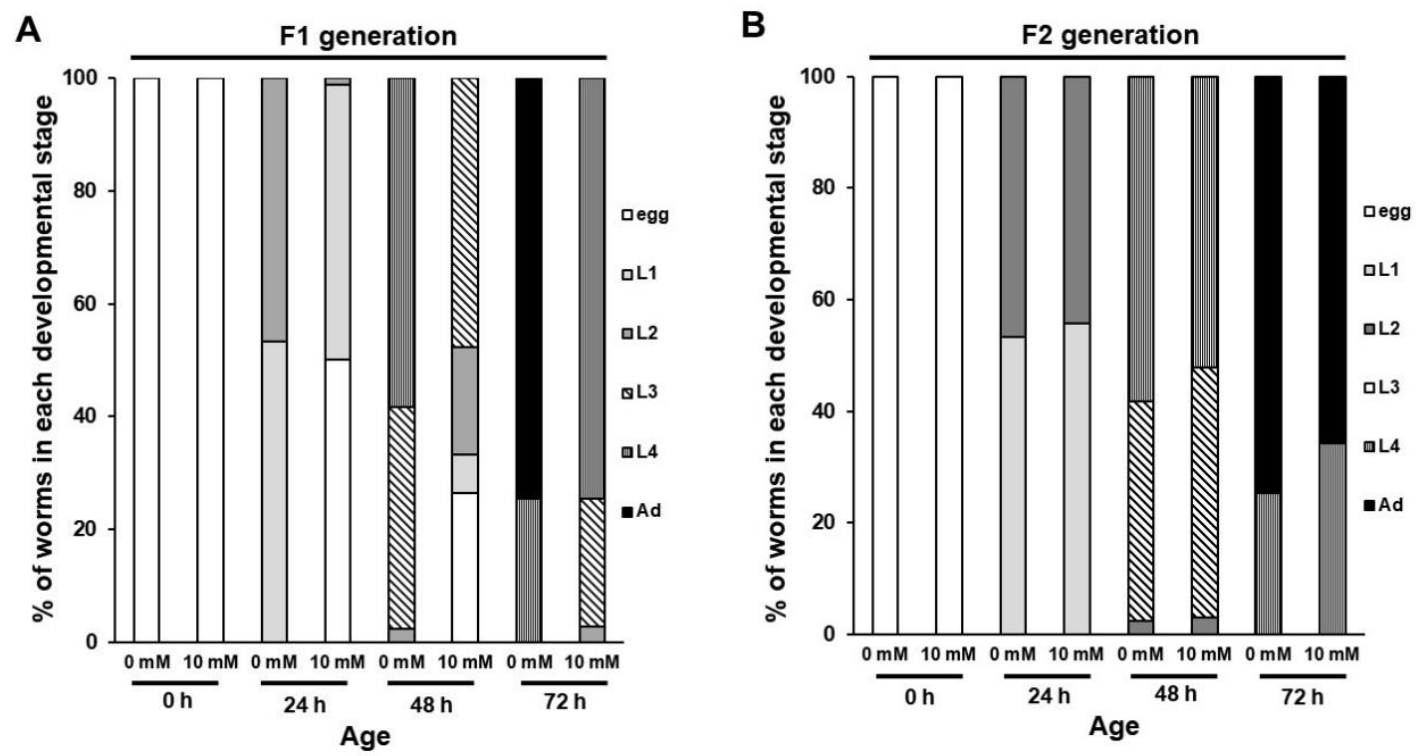

Figure 3. Caffeine intake by P0 mothers affected development in the subsequent generation of Caenorhabditis elegans. (A,B) Synchronized L4-stage animals $(n=30)$ of C. elegans wild-type were fed either 0 or $10 \mathrm{mM}$ of caffeine for $24 \mathrm{~h}$, and embryos were transferred to respective OP50-seeded nematode growth medium plates and further cultured at $20^{\circ} \mathrm{C}$. The developmental stage of each individual in the F1 and F2 generations was determined based on its size and stage-specific morphological characteristics (see Section 2 for details) during development by either caffeine-ingested $(10 \mathrm{mM})$ or caffeine-free diet P0 mother (0 mM).

\subsection{Maternal Caffeine Intake Reduces Yolk Production in C. elegans}

Vitellogenin (yolk proteins) plays an important role in the normal development of the animal's offspring by supplying nutrients [15-17]. To understand the molecular mechanism underlying the retarded growth rate of offspring produced by caffeine-ingested mothers, we investigated the possibility of association with the expression of vitellogenin. We measured the expression level of vitellogenin gene 6 (vit-6) and vitellogenin gene 2 (vit-2) after caffeine intake using transgenic animals with a transgene vit-6::gfp or vit-2::gfp. Adult-stage transgenic animals expressing either VIT-6::GFP or VIT-2::GFP were examined following exposure of caffeine and expression levels of both VIT-6::GFP and VIT-2::GFP were found to be reduced (Figure 4A-D). We confirmed a significantly reduced level of VIT-6::GFP compared to the control by Western blot analysis (Figure 4B). VIT-2 and VIT-6 are exclusively expressed in the intestine at the adult stage and VIT-2 is transported into oocytes and eventually to the embryonic cells, whereas VIT-6 remains in the intestine [18]. We thus observed oocytes and embryos for VIT-2::GFP expression and found a significantly decreased level of VIT-2::GFP both in oocytes and embryos after caffeine intake (Figure 4C,D). These results demonstrate that the expression of vitellogenin genes is suppressed by caffeine intake. 

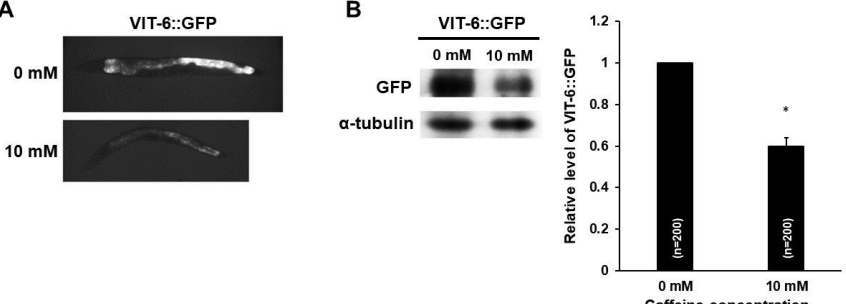

C
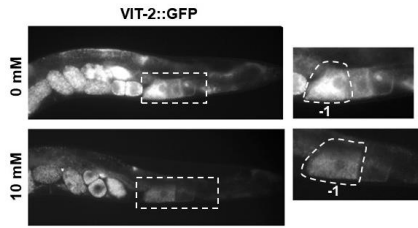

D
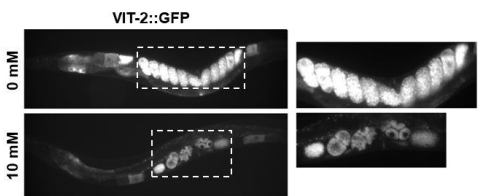
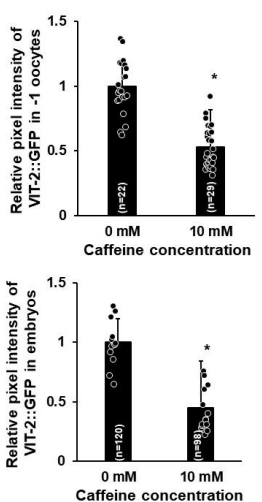

E

$\mathbf{F}$
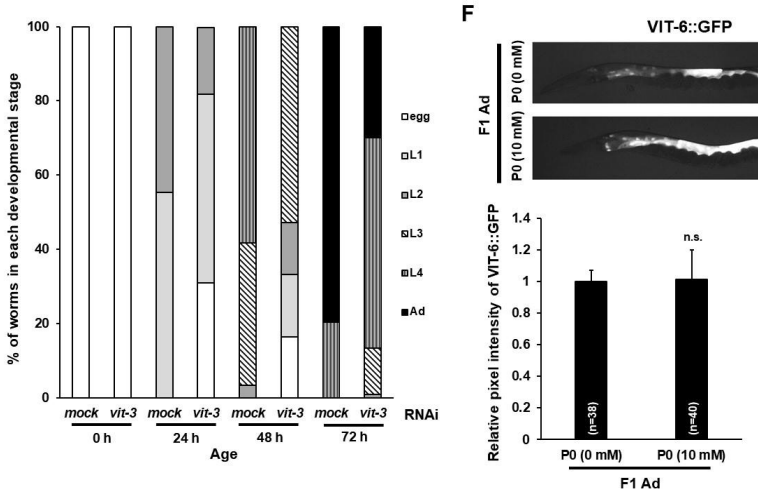

Figure 4. Maternal caffeine intake reduces vitellogenin (yolk proteins) in Caenorhabditis elegans. (A) VIT-6::GFP transgenic animals synchronized at the L4-stage were exposed to caffeine for $24 \mathrm{~h}$ at $20{ }^{\circ} \mathrm{C}$. VIT-6::GFP expresses in the intestine. The reduced level of VIT-6::GFP in the intestine was observed in caffeine-ingested mothers. (B) Western blot analysis of VIT-6::GFP protein levels in caffeine-free diet mother $(0 \mathrm{mM})$ and caffeine-ingested mother $(10 \mathrm{mM})$. Respective GFP band intensities were normalized against those of $\alpha$-tubulin in the same lane. Then the normalized GFP band intensity was converted to a relative value compared to the normalized GFP band intensity of $0 \mathrm{mM}$, as shown in the right graph with mean \pm SD values. These GFP band intensity values were obtained from three independent Western blot analyses. Statistical significance was calculated using Student's $t$-test. ${ }^{*} p<$ 0.05. (C,D) VIT-2::GFP transgenic animals synchronized at the L4-stage were exposed to caffeine for $24 \mathrm{~h}$ at $20^{\circ} \mathrm{C}$. The caffeine-ingested mother showed a reduced level of VIT-2::GFP intensity in oocytes and embryos, as shown in the right graph with mean \pm SD values. (E) Synchronized L4-stage animals $(n=30)$ of wild-type were treated with RNA interference (RNAi) of vit-3 gene for $24 \mathrm{~h}$ and recovered to OP50-seeded nematode growth medium (NGM) plates for $24 \mathrm{~h}$. The embryos produced by P0 mothers were transferred to respective NGM plates and further cultured at $20^{\circ} \mathrm{C}$. The developmental stage of each individual in the progenies was determined based on its size and stage-specific morphological characteristics (see Section 2 for details) during development by either the vit-3 RNAi-treated P0 mother $(10 \mathrm{mM})$ or non-treated (mock) P0 mother $(0 \mathrm{mM})$. (F) VIT-6::GFP transgenic animals synchronized at the L4-stage were exposed to caffeine for $24 \mathrm{~h}$ at $20^{\circ} \mathrm{C}$. Then their $\mathrm{F} 1$ generation grew under the caffeine-free diet $(0 \mathrm{mM})$ condition, and VIT-6::GFP transgenic F1 animals were observed at the adult stage. Statistical significance was calculated using Student's $t$-test. n.s., $p>0.05$. 
We further assessed a possible association between the reduction in vitellogenin and developmental growth of offspring by examining the developmental stages of $\mathrm{F} 1$ worms produced by $\mathrm{P} 0$ mothers treated with RNA interference (RNAi) of the vitellogenin gene vit-3. We observed a retarded developmental growth rate in vit-3 RNAi depleted worms (Figure 4E), indicating that the presence of vitellogenin is required for the normal growth of offspring. Taken together, these results suggest that caffeine intake by the mother reduces the production of vitellogenin, which causes retarded growth of offspring. We further examined whether the reduced level of vitellogenin observed in caffeine-ingested P0 mothers sustains in the adult-stage F1 offspring. Interestingly, F1 generation adults expressed VIT-6::GFP similarly to that in the caffeine-free diet control group (Figure 4F), suggesting that the effect of reduced levels of vitellogenin on the development of offspring is limited to F1 generation. These findings suggest that the decreased level of vitellogenin in the caffeine-ingested mother caused retarded larval growth of the F1 offspring, but the expression of vitellogenin in the adult stage of the F1 generation was not altered and no further effects were observed in F2 generation (Figures 3B and 4F).

\subsection{Maternal Caffeine Intake Disrupts Eggshell Integrity in C. elegans}

Caffeine-ingested mothers showed an increased level of embryonic lethality (Figure 2B). Chromosomal alterations in oocytes have been reported as the primary cause of embryonic lethality in C. elegans [19]. Therefore, we examined DNA morphology in -1 position oocytes of caffeine-ingested mothers under a fluorescent microscope after DNA staining with TO-PRO-3 fluorescent dye. The majority of mature oocytes in caffeine-ingested mothers contained six pairs of aligned and condensed chromosomes in their nuclei, which are characteristic of the diakinesis stage in meiotic prophase I in normal -1 position oocytes (Figure $5 \mathrm{~A}$ ). As little as $8.4 \%$ of oocytes showed a chromosomal abnormality, which was not statistically significant $(p=0.068)$. This result indicates that caffeine intake by mothers did not affect chromosomal integrity in oocytes.

The eggshell, which provides a protective structure with the extracellular matrix, is an important factor in many early developmental events $[13,20]$ and recent studies have suggested the pivotal role of the eggshell during embryonic development and survival [21,22]. Therefore, we next examined eggshell integrity in F1 embryos produced by caffeine-ingested mothers. We isolated embryos from dissected caffeine-ingested and caffeine-free diet mothers and examined their integrity. Approximately $17 \%$ of embryos isolated from caffeine-ingested mothers were ruptured (Figure $5 B, C$ ); the remaining embryos were examined with lipophilic dye FM4-64 staining for visualizing embryonic morphology $[13,23]$. Surprisingly, approximately $38 \%$ of the embryos out of the $83 \%$ non-ruptured embryos from caffeine-ingested mothers were permeable to FM4-64 and their embryonic membrane was stained, whereas embryos produced by caffeine-free diet mothers were not permeable to FM4-64 and their embryonic membrane was not stained at all (Figure 5D). Lipophilic FM4-64 dye binds to the embryonic membrane and stains it if the eggshell is disrupted. Taken together, maternal caffeine intake disrupted eggshell integrity, causing the eggshell to rupture and become permeable.

\subsection{Reduced Vitellogenin Production in Caffeine-Ingested Mothers Causes Defective Oocyte and Eggshell Integrity in C. elegans}

We found that caffeine intake reduced vitellogenin production (Figure 4A-D) and disrupted eggshell integrity (Figure 5B-D). On the basis of these findings, we investigated the relationship between vitellogenin and eggshell integrity to determine whether disrupted eggshell integrity could be due to the reduction in vitellogenin production. The vitellogenin family is comprised of six genes: vit-1 to vit-6 [24]. We performed the respective RNAi of the six vit genes to knock down vitellogenin production and observed eggshell permeability of embryos produced by RNAi-treated mothers (Figure 6A). Approximately 20\% of embryos were permeable to FM4-64 by depletion of vit genes, whereas a majority of mock RNAi-treated embryos were not permeable to FM4-64 (Figure 6A). The eggshell permeability defects in the vit RNAi experiments were likely due to simultaneous knockdown of more than one vit gene, due to the RNAi target sequence similarity to more than one vit gene. 
Off target effects likely occur because vit- 1 is $82 \%$ identical to vit-2, vit-3 and vit-4 are $99 \%$ identical, vit-5 is $96 \%$ identical to vit-3, and vit-6 is $50 \%$ identical to vit-2 [17]. Indeed, a single vit mutant only showed subtle phenotype, suggesting the functional redundancy among vit genes. We observed that approximately $10 \%$ of embryos in vit-1(ok2616) and vit-2(ok3211) mutants, and approximately $1.7 \%$ of embryos in vit-5(ok3239) mutant were permeable to FM4-64 dye (Figure 6B). Here we presented results from vit RNAi and mutant analyses (Figure 6), which suggest that the reduction in yolk proteins disrupts eggshell integrity.

A
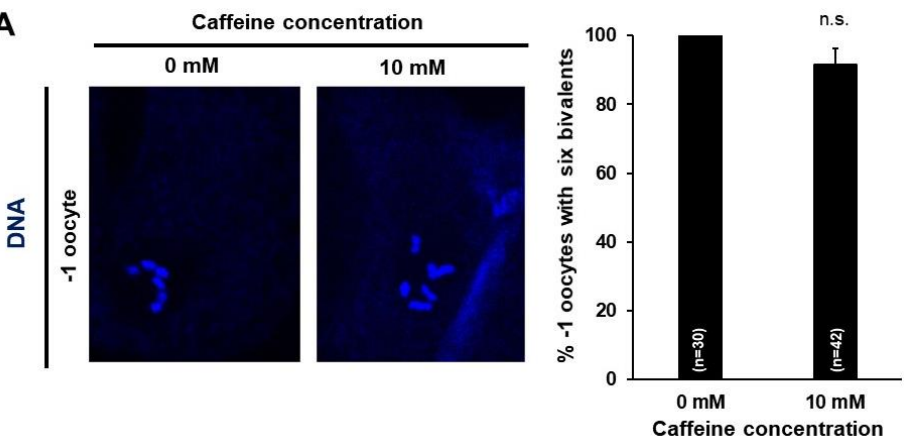

B
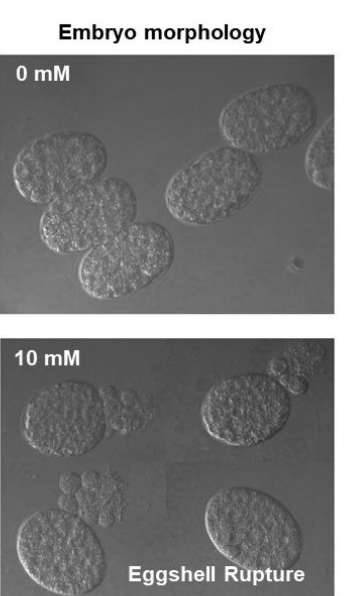

C
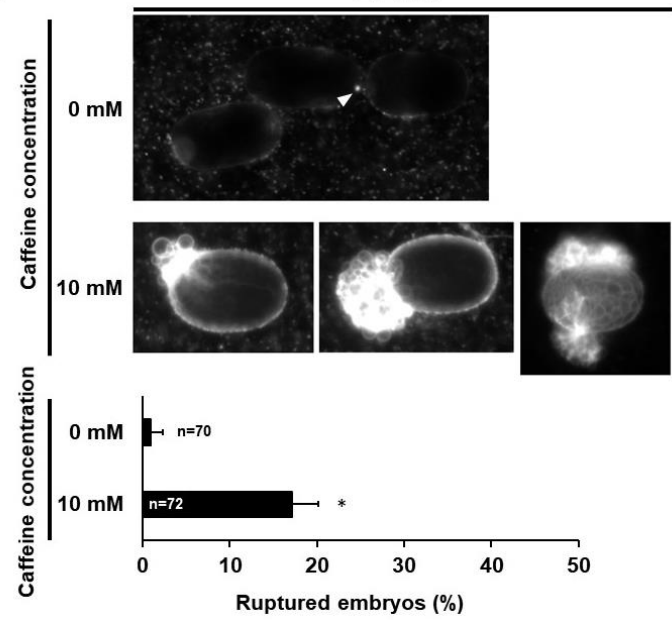

D

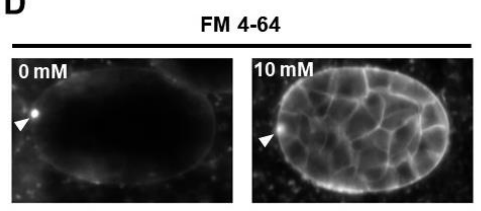

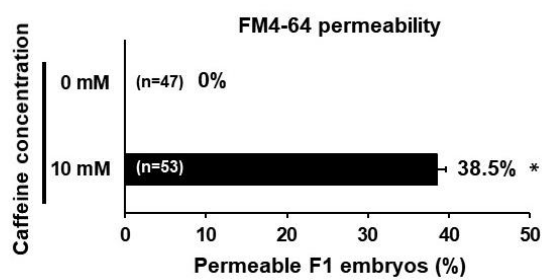

Figure 5. Maternal caffeine intake disrupted eggshell integrity in Caenorhabditis elegans. (A) Oocytes at the -1 position in a gonad arm of wild-type adult hermaphrodites grown without or with $10 \mathrm{mM}$ caffeine. DNA was stained with TO-PRO-3 and then six bivalents were examined. The percent of intact six bivalents is shown in a bar graph on the right. (B-D) DIC images (B) and lipophilic dye FM4-64 staining images (C,D) of embryos from the dissected caffeine-free diet $(0 \mathrm{mM})$ and caffeine-ingested mothers $(10 \mathrm{mM})$. The caffeine-free diet $(0 \mathrm{mM})$ mother produced intact and ovoid embryos and only polar bodies (white arrowheads) were stained, but the caffeine-ingested mother produced ruptured embryos (C) and their eggshells were permeable to FM4-64, and the cell membranes of the embryos were stained (D). Statistical significance was calculated using Student's $t$-test. ${ }^{*} p<0.05$. 
Next, we examined whether disrupted eggshell integrity is associated with oocytes in caffeine-ingested mothers. We hypothesized that disruption in eggshell integrity is possibly due to defective oocytes in caffeine-ingested mothers and thus we examined permeability of oocytes using lipophilic dye FM4-64. Interestingly, caffeine-ingested mothers exhibited approximately $20 \%$ of gonads with permeable oocytes, unlike oocytes produced by caffeine-free diet mothers (Figure 6C). Furthermore, we also performed RNAi of respective vitellogenin genes to test whether the reduction in vitellogenin can also allow oocytes to be permeable. The respective vitellogenin gene (vit-1, -2 , $-3,-4,-5$, and -6) RNAi-treated mothers produced approximately $18-22 \%$ of permeable oocytes in gonads (Figure 6D). These findings indicate that maternal caffeine intake caused defective oocytes and disrupted eggshell integrity through the reduction in vitellogenin production.

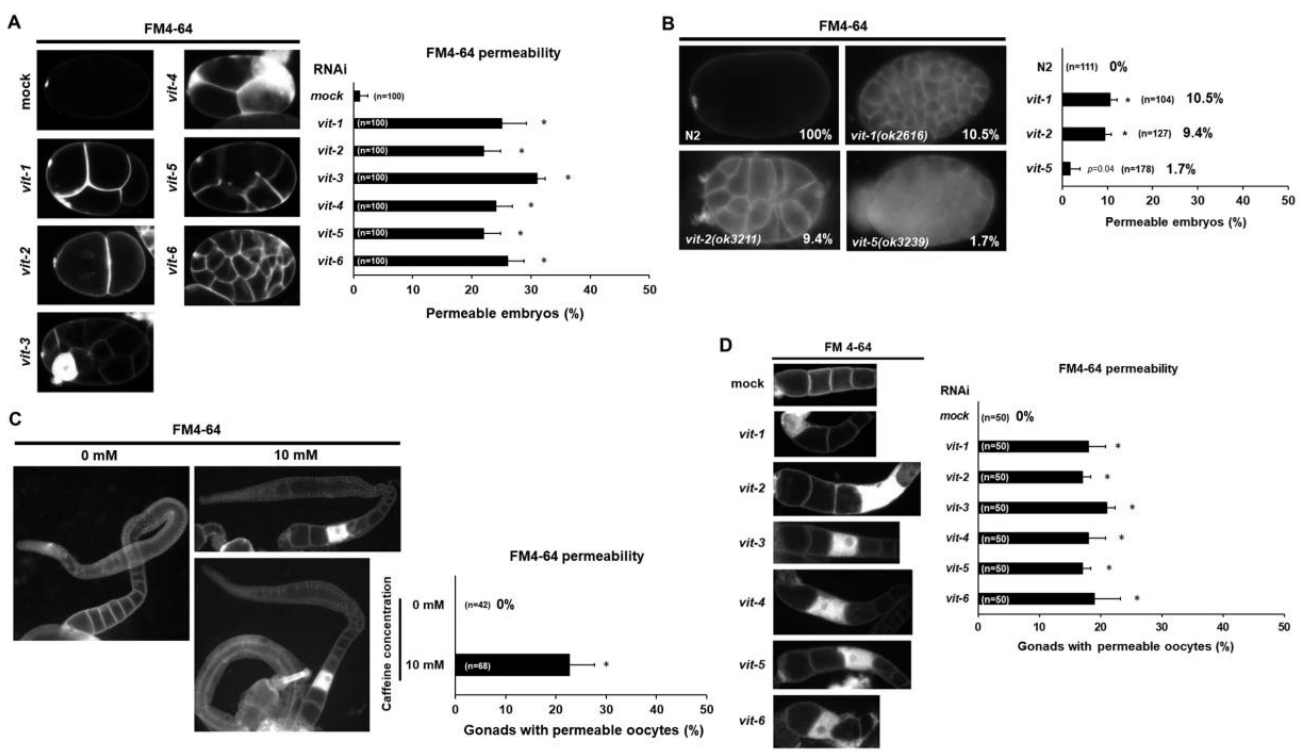

Figure 6. Vitellogenin is required for eggshell and oocyte integrity in Caenorhabditis elegans. (A) Eggshell permeability was examined by lipophilic dye FM4-64 staining in the embryos produced by mock RNAior vit-1 to vit-6 RNAi-treated mothers. Statistical significance was calculated using Student's $t$-test. * $p<0.05$ against mock RNAi-treated animals. (B) Eggshell permeability was examined by FM4-64 staining in the embryos produced by vit-1(ok2616), vit-2(ok3211), and vit-5(ok3239) mutants. Statistical significance was calculated using Student's $t$-test. ${ }^{*} p<0.05$ against N2 control. (C) Oocyte permeability examined with lipophilic dye FM4-64 staining in the caffeine-ingested mother (10 $\mathrm{mM})$ and caffeine-free diet mother $(0 \mathrm{mM})$. Gonads were extruded by dissecting adult animals in $150 \mathrm{mM}$ of $\mathrm{KCl}$. Statistical significance was calculated using Student's $t$-test. ${ }^{*} p<0.05$. (D) Oocyte permeability was examined by FM4-64 staining after treatment with RNAi of the vitellogenin genes from vit-1 to vit-6. Statistical significance was calculated using Student's $t$-test. ${ }^{*} p<0.05$ against mock RNAi-treated animals.

3.5. Maternal Caffeine Intake Reduces unc-62 Expression and the Reduced Level of unc-62 Causes Defective Oocyte and Eggshell Integrity in C. elegans

It has been reported that VPE1 (TGTCAAT) and VPE2 (CTGATAA), the cis-elements in the vit promoter, are important for vitellogenin (vit) gene expression (Figure 7A), [25,26]. VPE1 is bound by the UNC-62 (human Meis homeobox) transcription factor, which is highly expressed in the intestines of adult C. elegans, where vit genes are specifically expressed; and expression of vit genes is suppressed in unc-62 RNAi-treated animals [27]. Therefore, we investigated whether unc-62 expression is affected by caffeine intake by measuring the mRNA level of unc-62 gene in caffeine-ingested and caffeine-free diet mothers using qRT-PCR (Figure 7B). We found that the mRNA level of unc-62 gene significantly decreased in caffeine-ingested mothers (Figure 7B) and the reduced unc-62 level by RNAi led to a decreased level of VIT-2::GFP (Figure 7C). These results suggest that the reduced vitellogenin production by caffeine intake is caused by the decreased level of transactivator unc- 62 . 
A

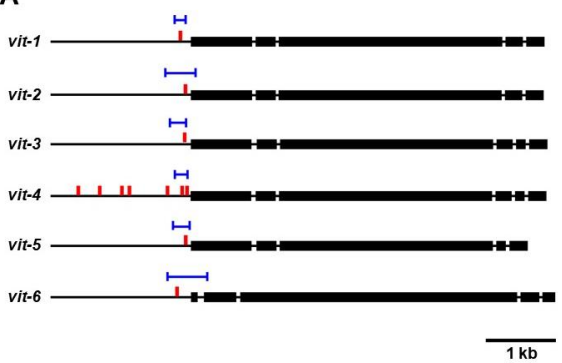

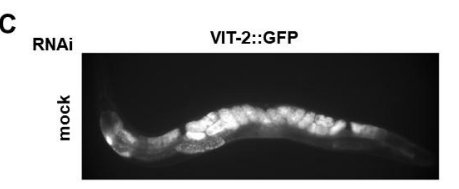

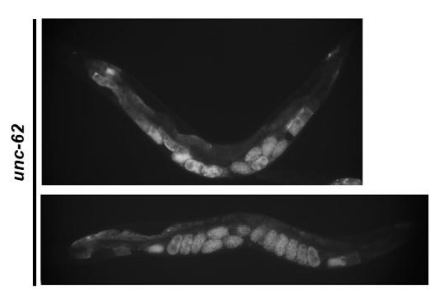

D
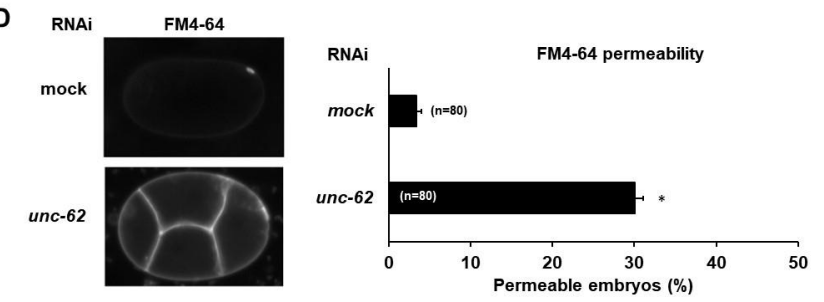

E

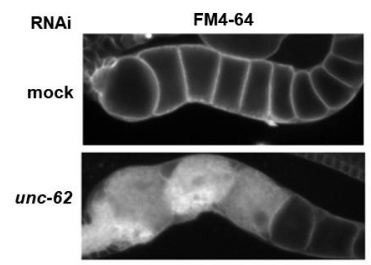

B
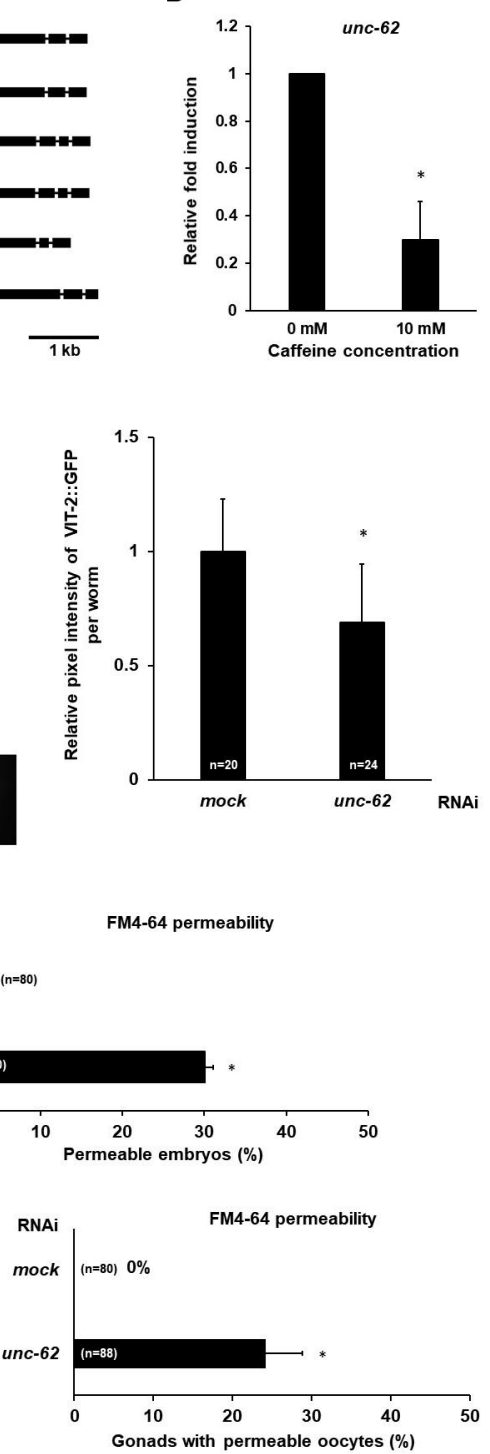

Figure 7. Caffeine intake reduces unc-62 expression and the reduced level of unc-62 exhibits defects in embryo and oocyte integrity in Caenorhabditis elegans. (A) UNC-62 binding sites in the genomic structures of vitellogenin genes. The thin black lines indicate the promoter and introns of each of six vitellogenin loci, and the thick black lines indicate exons of six vitellogenin genes. The blue lines indicate UNC-62 binding sites, and the red bars indicate VPE-1 (ATTGACA) vitellogenin regulatory motif previously described [25,27]. (B) Fold induction of mRNA level of unc-62 in caffeine-ingested mothers $(10 \mathrm{mM})$ than caffeine-free diet mothers $(0 \mathrm{mM})$. The mRNA level of unc-62 was determined by three independent qRT-PCR using the mRNA level of act-1 in each sample as an internal control for normalization. T-bars represent SD. Approximately 150 adult animal individuals were used to prepare total RNA for respective conditions. Statistical significance was calculated using Student's $t$-test. * $p<0.05$. (C) VIT-2::GFP transgenic animals synchronized at the L4-stage were treated with unc-62 RNAi. The unc-62 RNAi-treated mothers showed the reduced level of VIT-2::GFP intensity both in oocytes and embryos, as shown in the right graph with mean \pm SD values. (D) Eggshell permeability examined by lipophilic dye FM4-64 staining of the embryos produced by unc-62 RNAi-treated mothers. Statistical significance was calculated using Student's $t$-test. ${ }^{*} p<0.05$ against mock RNAi-treated animals. (E) Oocyte permeability examined by lipophilic dye FM4-64 staining in the dissected gonad from the unc-62 RNAi-treated mothers. Statistical significance was calculated using Student's $t$-test. $* p<0.05$ against mock RNAi-treated mothers. 
Next, we addressed whether the reduced level of unc-62 can cause defects in eggshell and oocyte integrity. We performed unc-62 RNAi in mothers and observed eggshell and oocyte integrity by FM4-64 dye staining. The unc-62 RNAi-treated embryos and oocytes also became permeable to FM4-64 dye (Figure 7C,D), indicating that the reduced level of unc-62 induced by caffeine intake results in defective eggshell and oocyte integrity possibly through the reduction in vitellogenin production. Taken together, we propose that maternal caffeine intake affects the survival and growth of offspring through the reduction in yolk protein production, which is mediated by the repression of unc- 62 gene expression (Figure 8).

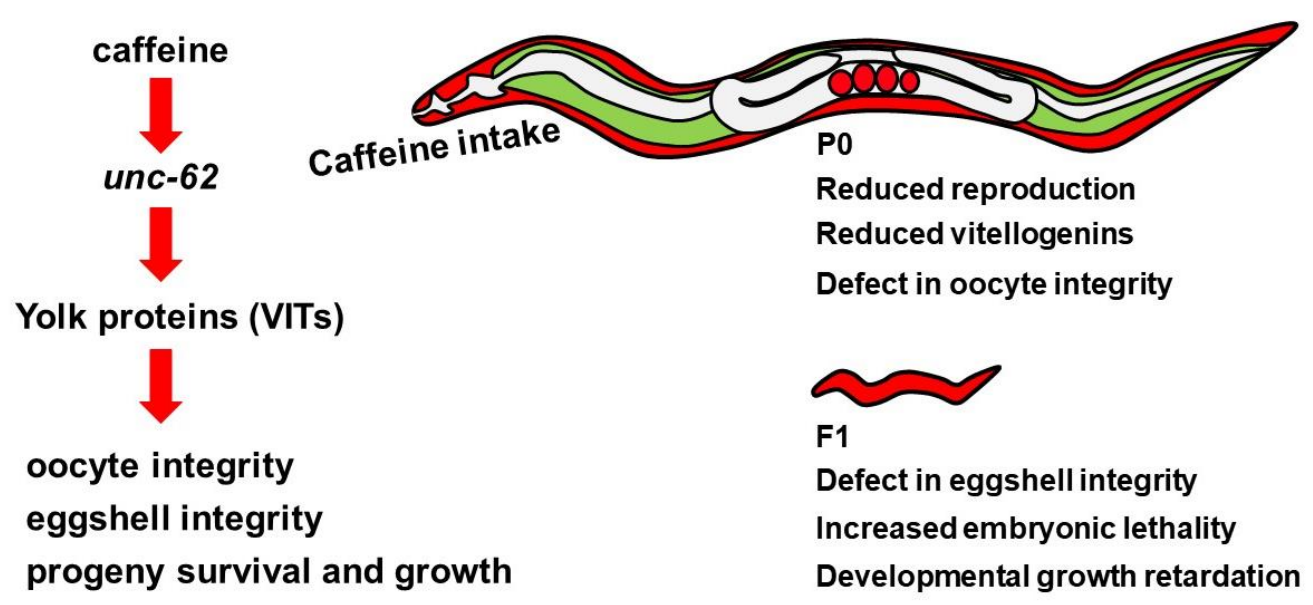

Figure 8. Model of the intergenerational effects of maternal caffeine intake in Caenorhabditis elegans. Caffeine intake decreased the production of yolk proteins by reducing unc-62 expression. The decreased levels of yolk proteins disrupted oocyte and eggshell integrity and induced embryonic lethality and growth retardation of the next generation.

\section{Discussion}

The physiological effects of caffeine intake in C. elegans are highly dose dependent [6]. Previous reports about the effects of caffeine using a $C$. elegans model indicate that the intake of a high dose of caffeine $(>10 \mathrm{mM})$ showed adverse effects, such as developmental arrest, activation of stress-response pathways, and stimulation of food-avoidance behavior [6-8], whereas animals treated with a low dose of caffeine $(<10 \mathrm{mM})$ generally showed beneficial effects such as lifespan extension, antioxidant effects, and protection of neurodegeneration $[10,28,29]$. However, the mechanism by which maternal caffeine intake affects not only the mother but also the offspring remains largely unknown. In this study, we examined the intergenerational effects of caffeine intake $(10 \mathrm{mM})$ by mothers on reproduction and offspring development in a C. elegans model. We found that $10 \mathrm{mM}$ of caffeine intake by the mother caused reduced fertility with defective oocytes and eggshell integrity, and an increased level of embryonic lethality and retardation in larval development (Figure 2A,B; Figure 3A).

There are six vit (vitellogenin) family genes and their expressions are stage-, sex-, and tissue-specific in C. elegans, which are exclusively and highly expressed in the adult hermaphrodite intestine [24]. Therefore, vit gene expression should be regulated by specific transcription factors. There are two major binding sites known as VPE1 and VPE2 in all six vit genes in C. elegans [25]. UNC-62, a VPE1-binding transcription factor, is highly expressed in the intestinal nuclei during the adult stage of C. elegans observed by UNC-62::GFP, and it activates vit gene expression [27]. unc-62 is an ortholog of human MEIS1 (Meis homeobox 1) and MEIS2 transcription factor that functions in normal human development [30]. In this study, we found that unc-62 expression was repressed by caffeine intake. How does caffeine intake regulate $u n c-62$ expression? It has been reported that caffeine intake influences metabolic rate and lipid oxidation [31-33]. In addition, it was suggested that nutrients can control transcription factors [34]. Since $u n c-62$ appears to be an upstream gene to control the developmental processes as a homeobox transactivator, this gene is possibly regulated by the nutritional state during 
the development of C. elegans. Considering that caffeine intake modulates the metabolic pathways and changes nutritional state in an animal, the developmental regulators are the possible targets responding to the alterations in metabolism. To investigate this possibility, the changes in the metabolic pathways in caffeine-ingested mother and the relation between these pathways and unc-62 repression needs to be determined. Vitellogenin (YP170) is transported from the intestine, where it is produced, to the oocytes by receptor-mediated endocytosis; it becomes enriched in the yolk and provides nutrients to the next generation in C. elegans [35]. Therefore, vitellogenin is considered as an intergenerational molecule that is transferred from the mother to the offspring. Suppression of vit gene expression after caffeine intake suggests that food signals are possibly involved in vitellogenesis. C. elegans vit genes are homologous to those in vertebrates [36]. In vertebrates, vitellogenin is produced in the female liver responding to estrogen signals and transported to the oocytes in the ovary through the blood. Furthermore, environmental estrogenic chemicals can induce vitellogenin production. Therefore, vitellogenin production is sensitive to the environmental status; thus, it can be used as a biomarker of environmental stress during reproduction [37]. The novel finding that vitellogenin is required for eggshell integrity and lack of vitellogenin causes embryonic lethality in approximately $20 \%$ of the embryos observed in this study suggests that the presence of vitellogenin is important for embryonic development of the next generation. Vitellogenin is mainly associated with lipid droplets in which phosphatidylcholine (PC, 23\%) and phosphoethanolamine (PE, 28.2\%) are over half of the total lipid content [17]. PC and PE are a major class of phospholipids that are the main constituents of the cell membrane [38]. Therefore, the reduction of vitellogenin along with PC and PE in oocytes may cause defects in the vitelline layer of oocytes and permeability. However, we propose that vitellogenin can partially contribute to the survival of embryos and other factors can compensate for the requirement of vitellogenin during embryogenesis. This is supported by the findings that $23 \%$ of live embryos were retained even with complete loss of vitellogenin in oocytes in rme-2 mutants [35]; and that vitellogenin production was remarkably decreased under dietary restriction in C. elegans, causing increased embryonic lethality that was suppressed by methionine supplement [39]. In addition, larval growth retardation in the F1 generation of caffeine-ingested mothers in the present study reveals that vitellogenin is indeed an intergenerational protein. A large amount of vitellogenin from the mother was observed in the early F1 larval stage of C. elegans, suggesting that maternal vitellogenin remains in the larval stage and provides nutrients for larval development in the F1 progeny [40]. However, in the present study, we found that the decreased level of vitellogenin in the mother affected the F1 generation, but not further generations, suggesting that it did not affect vitellogenin production in the adult-stage intestine and germ cells in the F1 generation.

The eggshell plays an important role in protecting the embryo by maintaining proper osmotic conditions and preventing the entry of potentially harmful molecules from the environment. In addition, the permeability barrier of the embryo also allows it to maintain the substances required for embryogenesis [41-43]. Previous studies have shown that fatty acid synthesis and modifications of enzymes are required for the formation of the permeability barrier in the embryo [23], and lipid metabolism is strongly associated with eggshell integrity [43]. It has been reported that in zebrafish, caffeine intake has a role in the suppression of fatty liver by downregulation of genes associated with lipogenesis, and enhancement of lipid oxidation and autophagy activity [44]. A relationship between caffeine intake and fat metabolism has also been observed in a rat model system, which has shown that caffeine intake reduces body fat by lipolysis and has an anti-obesity effect [45]. These findings suggest that caffeine intake affects lipid metabolism, which in turn possibly controls eggshell formation. Therefore, in addition to vitellogenin production, lipid metabolism appears to be involved in eggshell and oocyte integrity after caffeine intake. Further analysis of the direct relationship between caffeine intake and lipid metabolism in eggshell integrity remains to be determined. Dietary habit is one of the external stimulations to induce internal physiological effects. Caffeine intake can be an effective external stimulator. It has previously been described that caffeine intake causes global deacetylation of proteins and mimics caloric restriction through autophagy induction [46]. It will be worthwhile to 
examine whether the mode of action in oocyte and eggshell integrity after caffeine intake is related to autophagy.

In summary, our results suggest that caffeine intake by the mother affects reproduction in a dose-dependent manner. We demonstrated that maternal caffeine intake reduces yolk production by regulating unc-62 expression. The reduced vitellogenin production by caffeine intake, in turn, decreases embryonic survival by disrupting eggshell integrity, and inhibits larval development. As reported, vitellogenin expression is highly specific at the adult stage of $C$. elegans females. Therefore, further investigation of the consequences of caffeine intake in males to understand paternal effects is required.

\section{Conclusion}

This study provides several evidences showing the intergenerational effects of maternal caffeine intake. These effects were attributed to the suppression of yolk protein production mediated by a transcriptional activator, unc-62 (human Meis homeobox). These findings support that the mother's diet during pregnancy is critical for the survival and growth of progeny.

Author Contributions: H.M. and Y.-H.S. conceived and designed the study. H.M. and E.Y. conducted experiments. H.M., E.Y., and Y.-H.S. analyzed and interpreted the data. H.M. and Y.-H.S. wrote the manuscript. Y.-H.S. administrated the project and provided materials and reagents. All authors have read and agreed to the published version of the manuscript.

Funding: This study was supported by a grant from the Konkuk University in 2017 to Y.-H.S.

Acknowledgments: C. elegans strains were provided by the Caenorhabditis Genetics Center, which is funded by NIH Office of Research Infrastructure Programs (P40 OD010440). yk cDNA clones were provided by Yuji Kohara (National Institute of Genetics, Japan).

Conflicts of Interest: The authors declare no conflicts of interest.

\section{References}

1. Li, J.; Zhao, H.; Song, J.M.; Zhang, J.; Tang, Y.L.; Xin, C.M. A meta-analysis of risk of pregnancy loss and caffeine and coffee consumption during pregnancy. Int. J. Gynecol. Obestet. 2015, 130, 116-122. [CrossRef] [PubMed]

2. Papadopoulou, E.; Botton, J.; Brantsæter, A.L.; Haugen, M.; Alexander, J.; Meltzer, H.M.; Bacelis, J.; Elfvin, A.; Jacobsson, B.; Sengpiel, V. Maternal caffeine intake during pregnancy and childhood growth and overweight: Results from a large Norwegian prospective observational cohort study. BMJ Open 2018, 8, e018895. [CrossRef] [PubMed]

3. C. elegans Sequencing Consortium. Genome sequence of the nematode C. elegans: A platform for investigating biology. Science 1998, 282, 2012-2018. [CrossRef] [PubMed]

4. Calvo, D.R.; Martorell, P.; Genovés, S.; Gosálbez, L. Development of novel functional ingredients: Need for testing systems and solutions with Caenorhabditis elegans. Trends Food Sci. Technol. 2016, 54, 197-203. [CrossRef]

5. Kimble, J.; Crittenden, S.L. Controls of germline stem cells, entry into meiosis, and the sperm/oocyte decision in Caenorhabditis elegans. Annu. Rev. Cell Dev. Biol. 2007, 23, 405-433. [CrossRef] [PubMed]

6. Min, H.; Kawasaki, I.; Gong, J.; Shim, Y.H. Caffeine induces high expression of cyp-35A family genes and inhibits the early larval development in Caenorhabditis elegans. Mol. Cells 2015, 38, 236-242. [CrossRef] [PubMed]

7. Al-Amin, M.; Kawasaki, I.; Gong, J.; Shim, Y.H. Caffeine induces the stress response and up-regulates heat shock proteins in Caenorhabditis elegans. Mol. Cells 2016, 39, 163-168. [CrossRef]

8. Min, H.; Youn, E.; Kawasaki, I.; Shim, Y.H. Caffeine-induced food-avoidance behavior is mediated by neuroendocrine signals in Caenorhabditis elegans. BMB Rep. 2017, 50, 31-36. [CrossRef]

9. Bridi, J.C.; Barros, A.G.; Sampaio, L.R.; Ferreira, J.C.; Antunes Soares, F.A.; Romano-Silva, M.A. Lifespan extension induced by caffeine in Caenorhabditis elegans is partially dependent on adenosine signaling. Front. Aging Neurosci. 2015, 57, 220. [CrossRef]

10. Li, H.; Roxo, M.; Cheng, X.; Zhang, S.; Cheng, H.; Wink, M. Pro-oxidant and lifespan extension effects of caffeine and related methylxanthines in Caenorhabditis elegans. Food Chem. X 2019, 1, 100005. [CrossRef] 
11. Kolahdouzan, M.; Hamadeh, M.J. The neuroprotective effects of caffeine in neurodegenerative diseases. CNS Neurosci. Ther. 2017, 23, 272-290. [CrossRef] [PubMed]

12. Brenner, S. The genetics of Caenorhabditis elegans. Genetics 1974, 77, 71-94. [PubMed]

13. Johnston, W.L.; Krizus, A.; Dennis, J.W. The eggshell is required for meiotic fidelity, polar-body extrusion and polarization of the C. elegans embryo. Genetics 2006, 4, 35. [CrossRef]

14. Min, H.; Kim, J.S.; Ahn, J.; Shim, Y.H. Gliadin intake causes disruption of the intestinal barrier and an increase in germ cell apoptosis in a Caenorhabditis elegans model. Nutrients 2019, 11, E2587. [CrossRef]

15. Wahli, W.; Dawid, I.B.; Ryffel, G.U.; Weber, R. Vitellogenesis and vitellogenin gene family. Science 1981, 212, 298-304. [CrossRef]

16. Carducci, F.; Biscotti, M.A.; Canapa, A. Vitellogenin gene family in vertebrates: Evolution and functions. Eur. Zool. J. 2019, 86, 233-240. [CrossRef]

17. Perez, M.F.; Lehner, B. Vitellogenins-Yolk gene function and regulation in Caenorhabditis elegans. Front. Physiol. 2019, 10, 1067. [CrossRef]

18. Hall, D.H.; Winfrey, V.P.; Blaeuer, G.; Hoffman, L.H.; Furuta, T.; Rose, K.L.; Hobert, O.; Greenstein, D. Ultrastructural features of the adult hermaphrodite gonad of Caenorhabditis elegans: Relations between the germ line and soma. Dev. Biol. 1999, 212, 101-123. [CrossRef]

19. Min, H.; Sung, M.; Son, M.; Kawasaki, I.; Shim, Y.H. Transgenerational effects of proton beam irradiation on Caenorhabditis elegans germline apoptosis. Biochem. Biophys. Res. Commun. 2017, 490, 608-615. [CrossRef]

20. Stein, K.K.; Golden, A. The C. elegans eggshell. WormBook 2018. Available online: http://www.wormbook.org (accessed on 2 August 2018).

21. Johnston, W.L.; Dennis, J.W. The eggshell in the C. elegans oocyte-to-embryo transition. Genesis 2012, 50, 333-349. [CrossRef]

22. Fickentscher, R.; Struntz, P.; Weiss, M. Mechanical cues in the early embryogenesis of Caenorhabditis elegans. Biophys. J. 2013, 103, 1805-1811. [CrossRef] [PubMed]

23. Olson, S.K.; Greenan, G.; Desai, A.; Müller-Reichert, T.; Oegema, K. Hierarchical assembly of the eggshell and permeability barrier in C. elegans. J. Cell Biol. 2012, 198, 731-748. [CrossRef] [PubMed]

24. Spieth, J.; MacMorris, M.; Broverman, S.; Greenspoon, S.; Blumenthal, T. Regulated expression of a vitellogenin fusion gene in transgenic nematodes. Dev. Biol. 1988, 130, 285-293. [CrossRef]

25. MacMorris, M.; Broverman, S.; Greenspoon, S.; Lea, K.; Madej, C.; Blumenthal, T.; Spieth, J. Regulation of vitellogenin gene expression in transgenic Caenorhabditis elegans: Short sequences required for activation of the vit-2 promoter. Mol. Cell Biol. 1992, 12, 1652-1662. [CrossRef] [PubMed]

26. Goszczynski, B.; Captan, V.V.; Danielson, A.M.; Lancaster, B.R.; McGhee, J.D. A 44bpintestine-specific hermaphrodite-specific enhancer from the $C$. elegans vit-2 vitellogenin gene is directly regulated by ELT-2,MAB-3, FKH-9 and DAF-16 and indirectly regulated by the germline, by daf-2/ insulin signaling and by the TGF- $\beta /$ Sma/Mab pathway. Dev. Biol. 2016, 413, 112-127. [CrossRef] [PubMed]

27. Van Nostrand, E.L.; Sánchez-Blance, A.; Wu, B.; Nguyen, A.; Kim, S.K. Roles of the developmental regulator unc-62/ Homothorax in limiting longevity in Caenorhabditis elegans. PLoS Genet. 2013, 9, e1003325. [CrossRef] [PubMed]

28. Sutphin, G.L.; Bishop, E.; Yanos, M.E.; Moller, R.M.; Kaeberlein, M. Caffeine extends life span, improves healthspan, and delays age-associated pathology in Caenorhabditis elegans. Longev. Healthsapn. 2012, 1, 9. [CrossRef]

29. Manalo, R.V.M.; Medina, P.M.B. Caffeine protects dopaminergic neurons from dopamine-induced neurodegeneration via synergistic adenosine-dopamine D2-like receptor interactions in transgenic Caenorhabditis elegans. Front. Neurosci. 2018, 12, 137. [CrossRef]

30. Moskow, J.J.; Bullrich, F.; Huebner, K.; Daar, I.O.; Buchberg, A.M. Meis1, a PBX1-related homeobox gene involved in myeloid leukemia in BXH-2 mice. Mol. Cell Biol. 1995, 15, 5434-5443. [CrossRef]

31. Acheson, K.J.; Zahorska-Markiewicz, B.; Pittet, P.; Anantharaman, K.; Jéquier, E. Caffeine and coffee: Their influence on metabolic rate and substrate utilization in normal weight and obese individuals. Am. J. Clin. Nutr. 1980, 33, 989-997. [CrossRef]

32. Bracco, D.; Ferrarra, J.M.; Arnaud, M.J.; Jéquier, E.; Schutz, Y. Effects of caffeine on energy metabolism, heart rate, and methylxanthine metabolism in lean and obese women. Am. J. Physiol. 1995, 269, E671-E678. [CrossRef] [PubMed] 
33. Acheson, K.J.; Gremaud, G.; Meirim, I.; Montigon, F.; Krebs, Y.; Fay, L.B.; Gay, L.J.; Schneiter, P.; Schindler, C.; Tappy, L. Metabolic effects of caffeine in humans: Lipid oxidation or futile cycling? Am. J. Clin. Nutr. 2004, 79, 40-46. [CrossRef] [PubMed]

34. Hanson, R.W. Nutrient control of gene transcription minireview series. J. Biol. Chem. 2000, $275,30747$. [CrossRef]

35. Grant, B.; Hirsh, D. Receptor-mediated endocytosis in the Caenorhabditis elegans oocyte. Mol. Biol. Cell 1999, 10, 4311-4326. [CrossRef]

36. Spieth, J.; Blumenthal, T. The Caenorhabditis elegans vitellogenin gene family includes a gene encoding a distantly related protein. Mol. Cell Biol. 1985, 5, 2495-2501. [CrossRef]

37. Denslow, N.D.; Chow, M.C.; Kroll, K.J.; Green, L. Vitellogenin as a biomarker of exposure for estrogen mimics. Ecotoxicology 1999, 8, 385-398. [CrossRef]

38. van der Veen, J.N.; Kennelly, J.P.; Wan, S.; Vance, J.E.; Vance, D.E.; Jacobs, R.L. The critical role of phosphatidylcholine and phosphatidylethanolamine metabolism in health and disease. Biochim. Biophys. Acta Biomembr. 2017, 1859, 1558-1572. [CrossRef]

39. Zhou, G.; Huang, C.; Xing, L.; Li, L.; Jiang, Y.; Wei, Y. Methionine increases yolk production to offset the negative effect of caloric restriction on reproduction without affecting longevity in C. elegans. Aging 2020, 12, 2680-2697. [CrossRef]

40. Kimble, J.; Sharrock, W.J. Tissue-specific synthesis of yolk proteins in Caenorhabditis elegans. Dev. Biol. 1983, 96, 189-196. [CrossRef]

41. Schierenberg, E.; Junkersdorf, B. The role of eggshell and underlying vitelline membrane for normal pattern formation in the early C. elegans embryo. Roux. Arch. Dev. Biol. 1992, 202, 10-16. [CrossRef]

42. Lee, J.Y.; Goldstein, B. Mechanisms of cell positioning during C. elegans gastrulation. Development 2003, 130, 307-320. [CrossRef] [PubMed]

43. Carvalho, A.; Olson, S.K.; Gutierrez, E.; Zhang, K.; Noble, L.B.; Zanin, E.; Desai, A.; Groisman, A.; Oegema, K. Acute drug treatment in the early C. elegans embryo. PLoS ONE 2011, 6, e24656. [CrossRef] [PubMed]

44. Zheng, X.; Dai, W.; Chen, X.; Wang, K.; Zhang, W.; Liu, L.; Hou, J. Caffeine reduces hepatic lipid accumulation through regulation of lipogenesis and ER stress in zebrafish larvae. J. Biomed. Sci. 2015, 22, 105. [CrossRef] [PubMed]

45. Kobayashi-Hattori, K.; Mogi, A.; Matsumoto, Y.; Takita, T. Effect of caffeine on the body fat and lipid metabolism of rats fed on a high-fat diet. Biosci. Biotechnol. Biochem. 2005, 69, 2219-2223. [CrossRef]

46. Pietrocola, F.; Malik, S.A.; Mariño, G.; Vacchelli, E.; Senovilla, L.; Chaba, K.; Niso-Santano, M.; Maiuri, M.C.; Madeo, F.; Kroemer, G. Coffee induces autophagy in vivo. Cell Cycle 2014, 13, 1987-1994. [CrossRef] 